\title{
QUALIDADE DEVIDA DE PACIENTES ACOMETIDOS DE DERMATOSES
}

\author{
*Felipe Fernandes Oliveira I \\ Luciana Cavalcante Trindade II \\ Natália Gontijo Ribeiro III \\ Stephany Pessoa Farias III \\ Kamila Paschoal Magno do Nascimento "I
}

\section{RESUMO}

O impacto psicológico gerado por uma dermatose deve ser cuidadosamente avaliado, pois poderá influenciar na evolução da doença e na resposta terapêutica. Nesse contexto, a presente investigação objetiva avaliar a qualidade de vida de pacientes com diferentes dermatoses. Trata-se de um estudo transversal cuja população foi composta por pacientes que, no período de novembro/18 a março/19, buscaram atendimento dermatológico em uma clínica-escola, localizada em João Pessoa/PB, utilizandose como instrumento o Índice de Qualidade de Vida em Dermatologia - DLQI. A associação entre variáveis qualitativas foi avaliada pelo teste de Qui-quadrado. Já para variáveis quantitativas foram utilizados testes não paramétricos. Para se comparar grupos de doenças foi utilizado o teste de Kruskal-Wallis, seguido do teste de comparações múltiplas de Dunn. As variáveis foram comparadas em relação ao sexo utilizando o teste $U$ de Mann-Whitney. Todos os aspectos éticos foram respeitados. Avaliaram-se 165 pacientes. A população foi composta por mulheres (70,3\%), com média de idade 35,25 $\pm 15,7$ anos. O tempo médio de doença foi de $3,6 \pm 4,0$ anos. As dermatoses mais prevalentes foram acne $(24,2 \%)$, micoses $(13,3 \%)$, tricoses $(12,7 \%)$, melasma $(7,9 \%)$, tumores cutâneos $(6,1 \%)$. A mediana do DLQI total $6,0(0-27,0)$ e média do escore geral foi de $7,62 \pm 6,15$, mostrando efeito moderado das dermatoses na qualidade de vida. A acne foi a dermatose que demonstrou pior dano a qualidade de vida. A análise alerta para uma avaliação abrangente do paciente que permita compreender melhor o quanto e de que forma a doença dermatológica pode prejudicar sua qualidade de vida e saúde mental.

PALAVRAS-CHAVE: Qualidade de Vida. Dermatopatia. Acne Vulgar.

Médico. Faculdade de Medicina Nova Esperança, FAMENE. Departamento de Medicina. CEP 58067695, João Pessoa, Paraíba, Brasil. *Autor Correspondente: fellipe_o@hotmail.com. Orcid: : 0000-0002-2618-7710

Médica. Mestre. Faculdade de Medicina Nova Esperança, FAMENE. CEP 58067695, João Pessoa, Paraíba, Brasil. Orcid: 0000-0002-0643-1093; 0000-0002-2793-0174

Médica. Faculdade de Medicina Nova Esperança, FAMENE. III CEP 58067695, João Pessoa, Paraíba, Brasil. Orcid: 0000-0001-6947-6910; 0000-0003-2751-8240 


\section{INTRODUÇÃO}

Qualidade de vida é um termo aberto a diversas interpretações, que envolvem temas amplos como bem-estar social, inserção do indivíduo na sociedade, saúde, família, estado emocional, entre outros. Essa expressão foi definida como "a percepção do indivíduo de sua posição na vida, no contexto da cultura e sistema de valores nos quais ele vive e em relação aos seus objetivos, expectativas, padrões e preocupações". ${ }^{1}$

Existe uma estreita ligação entre a pele e sistema nervoso central, uma vez que se originam da mesma camada de células germinativas, sendo muito atuais e promissores os temas relacionados a essa conexão. ${ }^{2,3} \mathrm{O}$ indivíduo com a pele comprometida, sobretudo em áreas descobertas, fica frequentemente envergonhado, ansioso ou triste. ${ }^{4}$

Estima-se que, pelo menos, um terço dos pacientes com doenças de pele tenha repercussões emocionais relacionadas à sua dermatose. $^{2}$ Portanto, torna-se relevante a avaliação do sofrimento dos pacientes relacionados às suas dermatoses e, também, a compreensão das alterações de qualidade de vida.5 Atualmente, muitas variáveis psicológicas são estudadas para uma melhor compreensão do adoecimento da pele. ${ }^{3}$ Alguns ensaios também procuram investigar intervenções que possam ser complementos dos tratamentos médicos, sendo a psicodermatologia o domínio de aplicação desses conhecimentos. ${ }^{4}$

A pele é intensamente afetada por tudo o que se passa na mente e, retrogradamente, desencadeia pensamentos e emoções pelo que se passa nela.4 Há permanente conexão mente- sistema nervoso-pele e uma relação recíproca entre essas três áreas, causando influência das doenças, tanto na saúde física, como sobre o desempenho no trabalho. As implicações na vida familiar, por sua vez, ampliaram o conceito do que é o tratamento das doenças. ${ }^{6}$

A vida dos pacientes é frequentemente afetada de forma profunda pela doença de pele, e o julgamento médico não se correlaciona bem com a percepção dos acometidos quanto à severidade da doença. ${ }^{7}$ Devido ao fato de se preocuparem com o que outras pessoas pensam de sua aparência, foi demonstrado também que as doenças de pele podem causar ansiedade, depressão, raiva e constrangimento, o que leva a isolamento social e absenteísmo no trabalho e na escola, levando a um alto grau de incapacidade, comparável a doenças crônicas graves. $^{8}$

As doenças de pele são as principais causas de morbidade na população geral devido a presença de sintomas físicos. Dessa forma, a medida do impacto subjetivo da doença para o paciente se torna particularmente importante. ${ }^{7,8}$ Os dermatologistas são responsáveis pela maioria dos atendimentos ambulatoriais de problemas psicocutâneos, no entanto, ensaios recentes sugerem que eles se sentem despreparados para lidar com a maior parte dos distúrbios psicodermatológicos.4 Assim, objetiva-se avaliar a qualidade de vida de pacientes portadores de diferentes dermatoses em uma clínica-escola localizada em João Pessoa, Paraíba, estabelecendo correlações entre as patologias e o grau de impacto na vida dos pacientes portadores. 


\section{MATERIAL E MÉTODOS}

Trata-se de um estudo transversal realizado com pacientes dermatológicos em uma clínica-escola, localizada em João Pessoa, Paraíba. A população foi composta por todos os pacientes que, no período de coleta dos dados, buscaram atendimento dermatológico na clínica-escola, contabilizando uma amostra de 165 pessoas. Os critérios de inclusão foram pacientes de qualquer sexo, etnia, com idade entre 18 e 75 anos, capazes de responder sobre o índice de qualidade de vida em dermatologia - DLQI, que procuraram o serviço por qualquer queixa dermatológica. Os meses de coleta foram de novembro de 2018 a março de 2019.

A todas as pessoas envolvidas na pesquisa foi previamente apresentado, lido e explicado o integral teor do Termo de Consentimento Livre e Esclarecido (TCLE). Tendo sido claramente orientados sobre a natureza da pesquisa para fins acadêmicos, razão pela qual muitos aceitaram participar livremente de forma gratuita e espontânea, demonstrando manifesta postura contributiva para a construção dos dados.

Após a consulta dermatológica de rotina, o pesquisador convidou os pacientes a participar da pesquisa com a aplicação do Índice de Qualidade de Vida para Dermatologia (DLQI), instrumento validado para uso no Brasil. $^{10} \mathrm{O}$ próprio paciente respondia ao questionário e entregava ao avaliador após término.

O DLQI consiste em dez itens divididos em seis categorias: 'sintomas e sentimentos', 'atividades diárias', 'lazer', 'trabalho/escola', 'relações interpessoais' e 'tratamento'. As respostas geram escores entre zero e três e o cálculo final é um somatório simples desses escores, com os índices maiores indicando pior qualidade de vida relacionada à doença. Os escores finais variam de 0 a 30: quanto maior o escore, pior a qualidade de vida. Um escore de 0-1 indica que não há impacto na vida do paciente; 2-5, o impacto é pequeno; 6-10, o impacto é moderado; 11-20, é grande impacto e, 21-30, impacto é extremo. ${ }^{8}$

As seguintes variáveis foram analisadas: dermatose, idade, sexo do paciente e tempo de evolução das doenças, sendo considerada significativa a associação para $p<0,05$. Para se avaliar a associação entre as variáveis qualitativas foi utilizado o teste de Qui-quadrado. Nas variáveis quantitativas (idade, tempo de doença e DLQI), foram utilizados testes não paramétricos, pois as variáveis não apresentaram aderência a curva normal (Teste de Kolmogorov-smirnov). Para se comparar os grupos de doenças foi utilizado o teste de Kruskal-Wallis, seguido do teste de comparações múltiplas de Dunn. $\mathrm{Na}$ comparação das variáveis com relação ao sexo ( 2 grupos independentes) foi utilizado o teste U de Mann-Whitney.

Para sua execução foi levado em consideração os aspectos éticos preconizados pela Resolução CNS 466/2012 e o Código de Ética Médica, Res. 1931/2009 CFM, Capítulo XII, que versa sobre ensino e pesquisa médica (CFM, 2010). Além disso, as identidades dos pacientes envolvidos na pesquisa são de total sigilo do pesquisador. $\mathrm{O}$ projeto foi aprovado pelo Comitê de Ética em Pesquisa da Faculdade de Medicina Nova Esperança (FAMENE) sob o CAAE 01557318.1.0000.5179. 


\section{RESULTADOS E DISCUSSÃO}

Para os 165 pacientes, avaliados no período do estudo, foi constatado que o tempo médio de doença é de 3,6 \pm 4,0 anos. As dermatoses mais prevalentes entre esses pacientes foram acne $(24,2 \%)$, micoses $(13,3 \%)$, tricoses (12,7\%), melasma (7,9\%), tumores cutâneos (6,1\%), conforme mostra a Tabela 1.

TABELA 1: Dermatoses acometidas por pacientes atendidos em uma clínica-escola, no período de novembro de 2018 a março de 2019 ( $n=165)$. João Pessoa-PB, 2020.

\begin{tabular}{ccc}
\hline \hline Diagnóstico & N & $\%$ \\
\hline Outros diagnósticos menos frequentes $^{1}$ & 59 & 35,8 \\
Acne & 40 & 24,2 \\
Micoses & 22 & 13,3 \\
Tricoses $^{2}$ & 21 & 12,7 \\
Melasma & 13 & 7,9 \\
Tumores cutâneos & 10 & 6,1 \\
\hline Total & 165 & $\mathbf{1 0 0}$ \\
\hline
\end{tabular}

Nota: ${ }^{1}$ dermatite seborreica, vitiligo, psoríase, dermatite de contato, tumores benignos; zalopecia areata, alopecia androgenética, eflúvio telógeno

$\mathrm{Na}$ análise do Índice de Qualidade de Vida para Dermatologia (DLQI), verificouse que $80,6 \%$ dos pacientes relataram algum efeito da doença sobre a qualidade de vida, sendo que $31,5 \%$ demonstraram efeito grande ou extremo.

Quanto ao resultado do DLQI total, a mediana foi de $6,0(0-27,0)$ mostrando efeito moderado das dermatoses na qualidade de vida. A média do escore geral foi de 7,62 \pm
6,15 também evidenciando moderado efeito. Não houve efeito da doença dermatológica na qualidade de vida em 19,4\% dos doentes ( 32 pacientes). Nos demais pacientes, a dermatose provocou algum efeito, sendo que $24,9 \%$ deles apresentaram "pouco efeito", 24,2\%, "efeito moderado", 28,5\%, "grande efeito" e $3 \%$ obtiveram DLQI >20, ou seja, efeito extremo sobre a qualidade de vida (Tabela 2 ).

TABELA 2: Relação entre o Índice de Qualidade de Vida para Dermatologia (DLQI) e pacientes dermatológicos atendidos em uma clínica-escola no período de novembro de 2018 a março de 2019 (n=165). João Pessoa-PB, 2020.

\begin{tabular}{ccccccc}
\hline DLQI & $\begin{array}{c}\text { Sem } \\
\text { efeito }\end{array}$ & $\begin{array}{c}\text { Pequeno } \\
\text { efeito }\end{array}$ & $\begin{array}{c}\text { Moderado } \\
\text { efeito }\end{array}$ & $\begin{array}{c}\text { Grande } \\
\text { efeito }\end{array}$ & $\begin{array}{c}\text { Extremo } \\
\text { efeito }\end{array}$ & Total \\
\hline \hline Masculino & $7(14,3 \%)$ & $15(30,6 \%)$ & $15(30,6 \%)$ & $10(20,4 \%)$ & $2(4,1 \%)$ & $49(100,0 \%)$ \\
Feminino & $25(21,5 \%)$ & $26(22,5 \%)$ & $25(21,5 \%)$ & $37(31,9 \%)$ & $3(2,6 \%)$ & $116(100,0 \%)$ \\
\hline
\end{tabular}

Na Tabela 3, foi relacionado o DLQI com as doenças dermatológicas. Os pacientes relatam que a acne e micoses apresentam efeito moderado sobre a qualidade de vida, enquanto que os pacientes acometidos por tumores cutâneos, melasma e tricoses demonstraram que esses apresentam pequeno efeito sobre sua qualidade de vida. 
TABELA 3: Relação entre o Índice de Qualidade de Vida para Dermatologia (DLQI) e os tipos de dermatoses que afetam pacientes atendidos em uma clínica-escola no período de novembro de 2018 a março de 2019 ( $n=165)$. João Pessoa-PB, 2020.

\begin{tabular}{cc}
\hline Dermatose & DLQI \\
\hline Acne & 10,73 \\
Micoses & 8,64 \\
Outros diagnósticos menos frequentes & 7,41 \\
Tricoses & 5,29 \\
Tumores cutâneos & 4,10 \\
Melasma & 3,77 \\
\hline
\end{tabular}

A população foi composta predominantemente por mulheres (70,3\%), com média de idade $35,25 \pm 15,7$ anos. A acne foi a dermatose que levou ao maior dano a qualidade de vida entre as analisadas. É provável que o caráter recidivante e incapacitante possa ter contribuído para tal achado. Corroborando com Taborda et al. ${ }^{5}$, quando descreveram achados como ansiedade e depressão em pacientes portadores dessa dermatose.

Dentre os pacientes avaliados com acne, verificamos graus variáveis da dermatose, além de alguns pacientes estarem em vigência de tratamentos. Verificaramse que pacientes com maior quantidade de pápulas, pústulas e nódulos, sem estar em vigência de tratamento, apresentaram grande efeito na qualidade de vida, ao passo que os pacientes com menor quantidade de lesões e em acompanhamento dermatológico demonstraram efeito pequeno a moderado em sua qualidade de vida. Dados semelhantes foram encontrados por Chowdary et al. ${ }^{11}$, quando encontraram significativa associação entre a gravidade dos graus de acne e os escores do DLQI.

No que se refere ao melasma, pode ser avassalador para o paciente, dependendo da extensão, pois, apesar de não ser ameaçador à vida, é bastante inestética, como demonstrado por Ali et al. ${ }^{12}$ que observaram grande impacto da doença na qualidade de vida dos pacientes. No entanto, na presente pesquisa, a dermatose respondeu pela menor média do DLQI, fazendo crer que a maior parte dos pacientes estudados já estivesse em vigência de tratamento e com acometimento mínimo, o que fez com que o dado obtido entrasse em conflito com pesquisas anteriores.

Dos pacientes com tumores cutâneos, o baixo impacto na qualidade de vida pode estar relacionado a ausência de sintomatologia, além da suposição de que alguns deles ainda desconheciam a repercussão do diagnóstico na sua saúde ao responderem ao questionário.

O DLQI dos pacientes com tricoses mostrou impacto baixo na qualidade de vida desses pacientes, o que possivelmente teve relação com a pequena extensão do dano no couro cabeludo dos pacientes avaliados. Dados conflitantes com a literatura, como avaliou Katoulis e colaboradores ${ }^{13}$, demonstrando que as alopecias geram uma carga psicológica mais pesada, afetando a qualidade de vida severamente, principalmente nos pacientes com alopecia cicatricial.

Avaliando ainda quanto ao tempo de doença, verifica-se que as dermatoses de caráter crônico e recidivante, como dermatite seborreica, psoríase, acne e eczemas em geral, levam a uma perda da qualidade de vida mais significativa do que as demais. Pode-se supor que, devido ao fato dessas doenças 
determinarem maior dedicação e mudança no estilo de vida dos pacientes, elas levam a um maior impacto em sua vida.

Comparando a idade dos pacientes e relacionando as dermatoses, os pacientes com acne apresentaram idade menor do que aqueles com as demais dermatoses $(p<0,05)$.
Pacientes com tricoses eram mais jovens do que pacientes com micoses, ou tumores cutâneos $(p<0,05)$, enquanto aqueles com tumores cutâneos eram mais velhos do que pacientes com outros diagnósticos, micoses ou melasma $(p<0,05)$.

TABELA 4: Relação entre idade e as dermatoses que afetam pacientes atendidos no Centro de Saúde Nova Esperança no período de novembro de 2018 a março de 2019 (n=165). João Pessoa-PB, 2020.

\begin{tabular}{cc}
\hline Dermatose & $\begin{array}{c}\text { Idade (anos) } \\
\text { MÉDIA }\end{array}$ \\
\hline Tumores cutâneos & 58,80 \\
Micoses & 41,14 \\
Melasma & 39,23 \\
Outros diagnósticos menos frequentes & 36,71 \\
Tricoses & 32,9 \\
Acne & 24,05 \\
\hline
\end{tabular}

\section{CONCLUSÃO}

A pesquisa evidenciou que as dermatoses causam impacto na qualidade de vida na maioria dos pacientes, notadamente naqueles com acne.

A importância desse estudo devese ao alertar para uma avaliação abrangente do paciente que permita compreender melhor o quanto e de que formas a doença dermatológica pode prejudicar a qualidade de vida e saúde mental das pessoas. O impacto psicológico gerado pela dermatose deve ser cuidadosamente avaliado e tratado individualmente, pois, sem dúvida, influenciará a evolução da dermatose e a resposta terapêutica.

Por fim, ressalta-se que a limitação do trabalho está relacionada ao fato de que a coleta de dados foi realizada em ambulatórios gerais de dermatologia, onde a demanda é vasta, inclusive quanto a variação no grau de gravidade do problema de pele e o momento terapêutico, no qual se encontra o paciente.

\section{LIFE QUALITY OF PATIENTS AFFECTED BY DERMATOSIS}

\section{ABSTRACT}

The psychological impact generated by a dermatosis should be carefully evaluated as it may influence the disease's evolution and therapeutic response. In this context, the present investigation aims to 
evaluate the quality of life of patients with different dermatosis. This is a cross-sectional study of which population were patients that, from November/18 to March/19, sought dermatological care in a clinicschool located in João Pessoa/PB, using the Quality of Life Index in Dermatology - DLQI as an instrument. The association between qualitative variables was evaluated by the Chi-square test; for quantitative variables non-parametric tests were used. The Kruskal-Wallis test was used to compare groups of diseases, followed by the Dunn multiple comparisons test. The variables were compared in relation to gender using the Mann-Whitney $\mathrm{U}$ test. All ethical aspects were respected. 165 patients were evaluated. The population was composed of women (70.3\%), mean age 35.25 \pm 15.7 years. The mean time of illness was $3.6 \pm 4.0$ years. The most prevalent dermatoses were acne (24.2\%), mycosis (13.3\%), trichosis (12.7\%), melasma (7.9\%), skin tumors (6.1\%). The median of total DLQI was 6.0 (0-27.0) and the mean overall score was $7.62 \pm 6.15$, showing a moderate effect of dermatoses on quality of life. Acne was the dermatosis that showed the worst damage to quality of life. The analysis alerts to a comprehensive assessment of the patient to better understand how much and how dermatological disease can damage his quality of life and mental health.

KEYWORDS: Quality of Life. Skin Diseases. Acne Vulgaris.

\section{REFERÊNCIAS}

1. PEREIRA, EF; TEIXEIRA, CS; SANTOS A. Qualidade de vida: abordagens, conceitos e avaliação. Rev Bras Fís Esporte. 2012; 26(2): 24150.

2. Weber MB, Lorenzini $\mathrm{D}$, Reinehr $\mathrm{CPH}$, Lovato B. Avaliação da qualidade de vida dos pacientes pediátricos de um centro de referência em Dermatologia no Sul do Brasil. An Bras Dermatol 2012;87(5):697-702.

3. Ludwig MWB, Oliveira MSO, Muller MC, Moraes JD. Qualidade de vida e localização da lesão em pacientes dermatológicos. An Bras Dermatol. 2009;84(2):143-50.

4. Azambuja RD. A necessidade de cuidado conjunto de dermatologistas, psiquiatras e psicólogos em Psicodermatologia. An Bras Dermatol. 2017;92(1):60-8.

5. Taborda ML, Weber MB, Teixeira KAM, Lisboa AP, Welter EQ. Avaliação da qualidade de vida e do sofrimento psíquico de pacientes com diferentes dermatoses em um centro de referência em dermatologia no sul do país. An Bras Dermatol. 2010;85(1):52-6.
6. Weber MB, Mazzotti NG, Prati C, Cestari TF. Aferição da qualidade de vida na avaliação global do paciente dermatológico. Revista HCPA. 2006; 26(2):35-44.

7. Paula HR, Haddad A, Weiss MA, Dini GM, Ferreira LM. Tradução, adaptação cultural e validação do questionário de qualidade de vida norte-americano Skindex-29. An Bras Dermatol. 2014;89(4):600-07.

8. Tejada CS, Mendoza-Sassi RA, Almeida Jr HL, Figueiredo PN, Tejada VFS. Impact on the quality of life of dermatological patients in southern Brazil. An Bras Dermatol. 2011;86(6):1113-21.

9. Radtke MA, Schafer I, Gajur A, Langenbruch $\mathrm{A}$, Augustin M. Willingness-to-pay and quality of life in patients with vitiligo. Br J Dermatol. 2009; 161 p. 134-39.

10. Martins GA, Arruda L, Mugnaini AS. Validação de questionários de avaliação da qualidade de vida em pacientes de psoríase. An Bras Dermatol. 2004; 79(5): 521-35.

11. Chowdary NK, Prabhu SS, Shenoi SD, Nayak 
SUK. Quality of life in acne patients: A clinical and Dermatology Life Quality Index (DLQI) based crosssectional study. Journ of Pakist Assoc of Dermatol. 2018; 28(4): 415-19.

12. Ali R, Aman S, Nadeem M, Kazmi AH. Quality of life in patients of melasma. Journ of Pakist
Assoc of Dermatol. 2013; 23(2):143-48.

13. Katoulis AC, Christodoulou C, Liakou Ai, Kouris A, Korkoliakou P, Kaloudi E, et al. Quality of life and psychosocial impact of scarring and non-scarring alopecia in women. Journ of the Germ Soc of Dermatol. 2013; 2:137-41. 